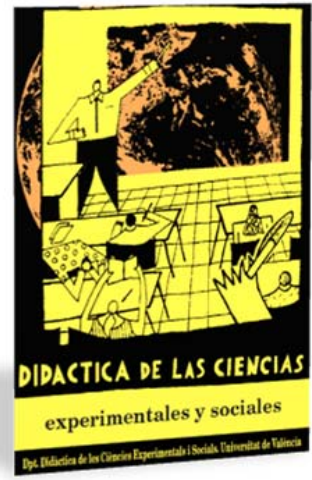

\title{
Medios de comunicación e Historia Reciente. Un estudio con alumnado chileno de secundaria
}

\author{
Mass Media and Recent History. A study with \\ Chilean secondary school students
}

DOI: $10.7203 / D C E S .34 .10516$

\author{
María Sánchez Agustí \\ Universidad de Valladolid \\ almagosa@sdcs.uva.es \\ Gabriela Vásquez Leyton \\ Pontificia Universidad Católica de Valparaíso \\ gabriela.vasquez@pucv.cl \\ Nelson Vásquez Lara \\ Pontificia Universidad Católica de Valparaíso \\ nelson.vasquez@pucv.cl
}

\begin{abstract}
RESUMEN: Se exponen parte de los resultados de una investigación cuyo objetivo es conocer las ideas de los estudiantes de secundaria chilena sobre el proceso Transición Democrática, enfatizando en las fuentes de conocimiento histórico que tienen sobre este tema. El estudio cuantitativo es realizado a partir de los discursos de 616 estudiantes de colegios privados, subvencionados y municipales de cuatro ciudades del país. Estas ideas se recogen a partir un cuestionario analizadas a través de un programa estadístico. Nuestros resultados indican que el caudal de conocimientos adquiridos por los estudiantes sobre la Transición no sólo proceden del ámbito escolar sino también, muy particularmente, de los medios de comunicación social, lo que debe ser considerado por el profesorado en su enseñanza.
\end{abstract}

Palabras clave: Medios de Comunicación, Concepciones de alumnos, Historia Reciente, Transición Dictadura-Democracia, Formación Ciudadana.

ABSTRACT: The aim of this paper is to analyse Chilean secondary-education students' ideas regarding this nation's transition to democracy, taking special interest in their sources of historical knowledge concerning this process. This study, which applies a mixed-methods approach, makes use of the narratives obtained from 616 students belonging to private, subsidised and municipal schools located in Chile's main urban areas. Students' perceptions are gathered by using a closed and open-ended questionnaire, and analysed by using a statistical computer software. The results show that, despite the high formative potential of history classes, the Chilean transition to democracy is not given a prominent importance in schools, leaving students no other choice than to resort to extracurricular sources to gain access to this knowledge.

KEYWORDS: mass media, students' perceptions, recent history, transition to democracy, citizenship education.

Fecha de recepción: julio de 2017

Fecha de aceptación: marzo de 2018

Este estudio forma parta del proyecto La Historia Reciente en la Educación. Diseño y evaluación de entornos digitales de aprendizaje en la enseñanza secundaria de España y Chile. EDU2013-43782-P del Plan Nacional I+D+I del MINECO (2013-2016), que representa la continuación del Proyecto I+D+i TRADDEC (EDU2009-09775) financiado por el Plan Nacional del MINECO de España (2010-2013). 


\section{INTRODUCCIÓN}

Desde mediados del siglo XX la enseñanza de la Historia ha consagrado oficialmente como objetivos centrales de su quehacer educativo, la tarea de enseñar a los estudiantes a pensar críticamente sobre el pasado y el presente, superando aquella visión más tradicional según la cual, la historia era una asignatura vinculada, principalmente, a la formación de los principios de la identidad nacional (Carretero y Borrelli, 2008). Esta situación ha permitido que, en los últimos años, el tema de la Historia Reciente, que anteriormente no tenía cabida en la escuela y no figuraba en los currículos escolares, hoy se vaya abriendo camino en las salas de clase de Historia, presentando desafíos y retos para la transposición didáctica de este conocimiento histórico.

En Chile, en los últimos años, se ha desarrollado una actualización del currículo nacional de la asignatura de Historia, Geografía y Ciencias Sociales (2009, 2013 y 2017), incorporándose contenidos asociados a los sucesos históricos más cercanos al presente en la Educación Media, situación que no presenta precedente curricular alguno y que tendrá profundas consecuencias en el sistema educativo, especialmente, en el desempeño del profesorado de la especialidad y en el aprendizaje histórico de nuestros estudiantes. Como producto de estas modificaciones curriculares se incorporan procesos históricos concretos de nuestro pasado reciente que buscan que los estudiantes aprendan hechos vinculados a la Historia de Chile del siglo XX, especialmente las tensiones y grandes quiebres vividos en los últimos años, con énfasis en la valoración de los derechos humanos, el respeto, la tolerancia y la democracia (Mineduc, 2013).

La elección representa un gran desafío para el profesor de Historia, por cuanto la incorporación de estas temáticas implica llevar a la escuela aquellos temas y problemas vinculados a procesos sociales conflictivos, traumáticos o controversiales, vividos por nuestro país en los últimos años (López Facal y Santidrán, 2011), precisamente aquellos sobre los cuales no hay consensos políticos y existe una notoria orfandad historiográfica, a diferencia de las visiones que han sido entregadas y profusamente difundidas por otras ciencias sociales, tales como la Sociología, la Ciencia Política y los medios de comunicación.

En este contexto, el presente artículo da cuenta de una investigación que ha pretendido analizar el rol que han cumplido los medios de comunicación en el conocimiento que poseen los estudiantes chilenos de segundo año de educación secundaria sobre la Transición desde la Dictadura a la Democracia. Nuestro interés radica en indagar en el tipo de medio de comunicación mayormente utilizado por el alumnado como principal fuente de información extraescolar (prensa, radio, televisión o internet) y, de esta manera, averiguar desde dónde aprendieron estos contenidos, cuáles son los referentes y los canales informativos que les han permitido construir su conocimiento, su pensamiento y su discurso narrativo sobre la temática histórica.

Hemos de tener en cuenta que las personas conocen la realidad que les circunda mediante explicaciones que extraen de los procesos de comunicación y del pensamiento social. Las representaciones sociales sintetizan dichas explicaciones y, en consecuencia, hacen referencia a un tipo específico de conocimiento que juega un papel crucial sobre cómo la gente piensa y organiza su vida cotidiana: el conocimiento del sentido común, el cual es influido culturalmente y tiene como particularidad la de ser socialmente construido y compartido en el seno de diferentes grupos.

Para Moscovici (1979, p. 18) "la representación social es un corpus organizado de conocimientos y una de las actividades psíquicas gracias a las cuales los hombres hacen inteligible la realidad física y social, se integran en un grupo o en una relación cotidiana de intercambios”, que se producen al interior de una sociedad. Es decir, son construcciones simbólicas que se crean y recrean en el curso de las interacciones sociales; no tienen un carácter estático ni determinan inexorablemente las representaciones individuales.

Por ello, en la sociedad actual, la circulación de ideas, imágenes y acontecimientos pasa por los medios masivos de comunicación, y resulta fundamental conocer y analizar las fuentes 
extraescolares que influyen en la construcción del conocimiento histórico del pasado reciente, pues determinan su visión de lo acontecido en el pasado y su proyección hacia el futuro.

\section{SOBRE LA HISTORIA RECIENTE Y SU ENSEÑANZA}

En los últimos años, el pasado cercano se ha constituido en objeto de interés por parte de una comunidad de historiadores que se sitúan en la llamada Historia Reciente o Historia del tiempo presente, motivación que se ha traducido en publicaciones, encuentros y congresos dedicados a problematizar la temática como un campo en construcción, logrando concatenar tres formas de relación entre el pasado y el presente. En primer lugar, la supervivencia de actores y protagonistas del pasado, en segundo, la existencia de una memoria social viva sobre ese pasado y, por último, la propia vivencia del historiador con aquel pasado del cual se ocupa. Aspecto importante lo constituye el periodo de tiempo que comprende esta Historia del pasado reciente, ya que el camino cronológico puede ser (aunque no el más pertinente) una opción para delimitar este campo de estudio. Sin embargo, en la comunidad de historiadores no existe acuerdo a la hora de establecer una cronología particular para este tipo historia, por lo tanto, los tres aspectos antes aludidos vendrían a constituir los elementos centrales para su estudio (Franco y Levín, 2007).

En ese sentido, una de las variables más importantes estará constituida por aquellos temas y problemas vinculados a procesos sociales conflictivos, traumáticos o controversiales, como guerras, genocidios, dictaduras y crisis sociales entre otras, que instituirán momentos de convulsión en los cuales se pone en riesgo y amenaza los lazos sociales, generando quiebres de difícil solución. Este tipo de acontecimientos son experimentados por los contemporáneos o coetáneos quienes los viven como momentos de crisis, rupturas, aspectos de continuidad y cambio, tanto desde el punto de vista individual como colectivo.

Ahora, tal como esbozamos anteriormente, si bien los diversos hechos traumáticos, constituyen un elemento central en la Historia Reciente, aquello no implica necesariamente la inauguración de una nueva época o periodo, como sucede con la historia contemporánea y sus orígenes vinculados a la revolución francesa, o con la historia presente y el término de la segunda guerra mundial (Aróstegui, 2004). En este aspecto, los hechos traumáticos de las dictaduras pueden ser significativos y marcan la conciencia de varias generaciones posteriores, porque resultan ser temas de difícil tratamiento, pero para que tenga sentido "no necesariamente inauguran nuevas épocas que pueden calificarse como recientes” (Figueroa e Iñigo, 2010: 26).

Aunque la Historia Reciente puede estar relacionada con una perspectiva universal o nacional, lo importante es vincular o articular ambos aspectos. Algo similar ocurre con la historia oral, la microhistoria y la nueva historia política que también han tenido una fuerte incidencia a la hora de abordar metodológicamente la historia del tiempo presente. Amén que también interesa prospectar la relación que se da entre historia reciente, memoria, testimonio y demanda social, porque tal como señala Rioux (1999) las preguntas que se plantea una sociedad en un momento determinado y que busca su respuesta en los historiadores, es una forma de conocer y comprender el pasado (inmediato) y divisar de mejor forma el porvenir.

En el caso chileno, para una transición hacia la democracia sin grandes estallidos sociales, la respuesta promovida por los gobiernos de la Concertación fue el olvido (Aceituno, 2013) o memoria del silencio (Carretero y Borrelli, 2008) respecto de los crímenes cometidos en dictadura. Pero a pesar de ello, las memorias resistieron, se fue aceptando de modo público el terror y las violaciones a los derechos humanos acontecidas - sobre todo desde 1998 con la agilización de los procesos judiciales -, y se generó más apertura en la discusión pública sobre el pasado.

Sin embargo, a pesar de ser la Historia Reciente algo que se aborda en los planes curriculares, estos temas no se presentan desde una perspectiva que promueva el debate (Toledo et al., 2015), sino que se fomenta una "memoria hegemónica", con el fin de excluir la memoria social y la reflexión crítica de los hechos históricos para imponer el consenso. Por ello, Carretero y Borrelli 
(2008) enfocan su análisis en el pasado reciente en interrelación de la dualidad Historia-Memoria, ya que consideran que la utilización de múltiples narrativas históricas entra en consonancia con lo expuesto por Kriger (2011) quien propone que la integración de "diversas memorias sociales" podría ser utilizado en el aula para promover investigaciones donde se utilice la metodología de la historia. Se fomentaría, así, el uso de fuentes diversas, lo que permitiría problematizar un tema en particular, contrastándolo con las informaciones presentadas tanto en los textos escolares como en los discursos oficiales.

Para Franco y Levín (2007) una decisión curricular que incorpore los procesos del pasado reciente, hace que la escuela deba legitimar su función educadora en ciertos valores éticos vinculados a la Democracia y, al mismo tiempo, construir cierta autoridad legítima por sobre las memorias múltiples de alumnos, padres y docentes. Tarea que, a no dudar, corresponde a las políticas educativas de cada país, al colaborar en la construcción de un relato legítimo de la Historia Reciente, que pueda ser transmitido por los profesores de la especialidad. Esto nos lleva a otro gran desafío, pues uno de los problemas reconocidos por la literatura es la falta de una cronología precisa que permita diferenciar qué es lo reciente de lo que no lo es. Sin embargo, lo cierto es que la historia reciente se caracteriza por la convivencia del pasado con el presente, la supervivencia de actores y protagonistas del pasado en condiciones de dar su testimonio de lo vivido, la existencia de una memoria social, la contemporaneidad entre la experiencia del historiador y el pasado del cual se ocupa. Situación que evidencia que estamos en frente de problemas y hechos ya pasados que se caracterizan por su presencia y actualidad en nuestro presente cotidiano como sociedad, como parece ser la decisión en la última actualización curricular.

En este contexto, analizar la llegada de la Historia Reciente a la escuela es una cuestión que refiere no sólo a las decisiones político-educativas o a sus reformulaciones didácticas sino también a las instituciones en su conjunto y a los profesores en particular. Fundamentalmente, porque como producto cultural y sociohistórico quienes enseñan historia tienen la alternativa de bucear en "las significaciones de los procesos históricos recientes, que como todos los transcursos se entraman en campos de fuerza de diferentes impulsos que operan en la cambiante intersección de discursos a menudo en conflicto" (Funes, 2006, p. 94).

Entendemos que las prácticas de enseñanza se moldean por la interacción de la propia biografía de los profesores, su experiencia personal y en el oficio docente, sus saberes, sus percepciones, su memoria, etc. Existe al mismo tiempo, una influencia importante de las tradiciones escolares y de los elementos contextuales amalgamados por la escuela como espacio cultural y como producto histórico, que puede promover o inhibir exigencias, expectativas, resistencias e incluso problemas que puede tener el profesorado para enfrentar el desafío de enseñar la historia del tiempo reciente. Un esfuerzo necesario y urgente es estudiar un actor clave en la superación de la fractura que en la actualidad existe entre la memoria del pasado que los hombres construyen para organizar sus vidas, estableciendo puentes desde la propia memoria personal y familiar hacia un pasado más amplio, construido con experiencias, recuerdos de gentes de otros momentos y de otras generaciones, lecturas, imágenes recibidas de los medios de comunicación, entre otros, y la historia que se enseña en las escuelas, que la gente común ve como un saber libresco (Fontana, 2001).

Es importante, pues, "tender puentes" entre los conocimientos que los estudiantes adquieren en el ámbito extraescolar y los proporcionados por el espacio formal de la escuela, donde los medios de comunicación se presentan con un potencial discursivo y de gran influencia dentro de la sociedad. Desde estos discursos se pueden basar los estudiantes a la hora de construir sus conocimientos previos sobre un contenido histórico determinado, más aún cuando se trata de los procesos propios de la Historia Reciente, cuyos temas, actores y acontecimientos son parte de la memoria viva de los contemporáneos y han encontrado un espacio propicios en los medios desde diversos ámbitos (documentales, cine de ficción, noticieros informativos, entre otros). Por ello, también es interesante enfatizar en el peso de los medios de comunicación en la construcción del conocimiento histórico de los docentes que enseñan historia escolar (Martínez Rodríguez, 2013), 
considerando que no podemos dejar de evidenciar que desde hace un tiempo, la prensa viene siendo utilizada en las aulas como procedimiento para la enseñanza de la Historia, como fuente histórica informativa. Esta situación nos permite un interesante vínculo desde el punto de vista didáctico, que debiese considerarse de manera integrada dentro de los conceptos propios del aprendizaje histórico (González Gallego, 1995).

Por lo anterior, hemos de considerar desde el ámbito didáctico que los medios de comunicación y las tecnologías digitales de la información conforman un sistema educativo informal que actúa como escuela paralela a la convencional con sus propios códigos, lenguajes, normas y valores (Aparici, 2005). Los medios de comunicación se han convertido en el corazón de la vida política y cultural de las personas, componiendo la imagen de una realidad que desean transmitir y, ante la dificultad, en muchos casos, de verificar las informaciones, sus receptores van integrándola como propia. De este modo, los medios influyen en la mentalidad de los jóvenes actuales, en su forma de atender y de captar su entorno social, político y cultural, en su actitud ante el conocimiento, en su modo de concebir el mundo e interactuar con su ambiente social (Liceras, 2005).

De ahí el interés de investigar sobre la incidencia de estos recursos extraescolares en los conocimientos históricos que los estudiantes aportan al aula. Este es el objetivo principal de este estudio en relación a un periodo histórico determinado: la Transición de la Dictadura a la Democracia. Coincidiendo con Miralles y Molina (2011), el análisis de los acontecimientos, fenómenos o realidades sociales recientemente producidos o que se encuentran en proceso de desarrollo, pueden convertirse en una interesante herramienta didáctica para la enseñanza de la Historia, a través de metodologías inductivas y de resolución de problemas que permitan la participación activa del alumnado en la construcción de su aprendizaje, así como convirtiendo la actualidad en la propia materia de estudio, haciendo de las problemáticas sociales más relevantes el eje temático a partir del cual planificar los contenidos, de manera que permitan el fortalecimiento de un pensamiento crítico en el alumnado, favorable para el desarrollo de una ciudadanía autónoma y responsable capaz de desenvolverse adecuadamente en una sociedad democrática.

Así mismo, la relevancia del tratamiento didáctico de los medios de comunicación de masas como fuentes de información es importante en la enseñanza, ya que buena parte de los lineamientos curriculares pretenden el conocimiento del mundo actual, tal como lo expresa el curriculum escolar chileno, con el fin de preparar a los jóvenes ciudadanos para la vida y dar claves para comprender el mundo en que viven y el entorno en el cual de desenvuelven. Para entender ese mundo, una de las mejores maneras es estudiarlo en sus problemáticas presentes y devenir inmediato (Hernández Cardona, 2002).

\section{SOBRE LA HISTORIA RECIENTE Y SU ENSEÑANZA}

Desde la estructura metodológica, este trabajo se enmarca dentro del ámbito de las Ciencias Sociales, siguiendo los lineamientos del paradigma interpretativo o hermenéutico, que pretende comprender la realidad, los significados y las intenciones de las personas con la finalidad de construir un nuevo conocimiento. El modelo enfatiza la interpretación de la realidad educativa, desde los significados de las personas implicadas en los contextos educativos (Arnal, 1992). Por tanto, el estudio que se presenta, tiene un carácter no experimental y se basa en la técnica de encuesta, mediante un cuestionario anónimo, aplicado en los centros educativos de manera directa por los investigadores, donde se recogen organizadamente los indicadores de las variables implicadas en el objetivo.

Este instrumento de recogida de información fue cumplimentado por 616 alumnos de tercer año de educación media (15-16 años), a partir de una muestra no probabilística, de conveniencia o por criterio de accesibilidad que consideró las cuatro ciudades con mayor población en Chile: La Serena, Viña del Mar, Santiago y Concepción (ver tabla $n^{0} 1$ ). Se escogió este nivel educativo 
porque, en el curso anterior, los estudiantes han trabajado los contenidos relacionados con la historia reciente chilena, donde se incluyen los temas relacionados con el golpe de estado, la dictadura y la transición democrática (Mineduc 2009) ${ }^{1}$.

TABLA 1. Muestra de la investigación

\begin{tabular}{|c|c|c|c|c|c|c|c|c|}
\hline & \multicolumn{2}{|c|}{ Particular } & \multicolumn{2}{|c|}{ Subvencionado } & \multicolumn{2}{|c|}{ Municipal } & \multirow{2}{*}{$\begin{array}{c}\text { Total } \\
\text { alumnos }\end{array}$} & \multirow[t]{2}{*}{ \%Participación } \\
\hline & $\mathrm{H}$ & MJ & $\mathrm{H}$ & MJ & $\mathrm{H}$ & MJ & & \\
\hline La Serena & 5 & 4 & 33 & 41 & 29 & 0 & 112 & $18,2 \%$ \\
\hline Valparaíso & 4 & 6 & 74 & 35 & 2 & 10 & 131 & $21,3 \%$ \\
\hline Santiago & 12 & 23 & 58 & 62 & 1 & 37 & 193 & $31,3 \%$ \\
\hline Concepción & 18 & 15 & 35 & 46 & 46 & 20 & 180 & $29,2 \%$ \\
\hline Total & 39 & 48 & 200 & 184 & 78 & 67 & 616 & \\
\hline \% particip. & $6,3 \%$ & $7,8 \%$ & $32,5 \%$ & $29,9 \%$ & $12,7 \%$ & & & $100 \%$ \\
\hline
\end{tabular}

Para el estudio se incluyeron establecimientos educativos de distinta tipología socioeconómica ${ }^{2}$ : Particular (CP), Subvencionado (CS) y Municipal (LM). La razón de esta selección radica en que el Informe de la Comisión de Formación Ciudadana (2004) desarrollado por el Ministerio de Educación de Chile y que buscaba proponer una visión sobre los desafíos que implica vivir en una sociedad democrática, consideró que el sistema educacional chileno presenta fuertes desigualdades de oportunidades y segmentación social. Pese a que los niveles de cobertura son altos, los decídeles de calidad con equidad son muy dispares, situación que incide inevitablemente en la calidad de la vida cívica en Chile así como en la formación democrática y ciudadana de los educandos (Mineduc, 2005).

Los resultados que aquí presentamos forman parte de una investigación más amplia, cuyo objetivo consiste en comprender las representaciones sociales de los estudiantes de secundaria chilenos sobre la transición de la dictadura a la democracia en Chile, así como la valoración que hacen de este contenido escolar para su formación democrática y ciudadana. Para los fines de este artículo, se ha escogido la pregunta referida a las fuentes de información que tienen los estudiantes para conocer el tema de la Transición de una dictadura a una democracia. Esta cuestión se presenta con la finalidad de averiguar cuáles son los referentes y los canales informativos que les han permitido construir su conocimiento y pensamiento sobre la temática histórica, tanto al interior de la sala de clases como fuera de ella. En éste aspecto, tomamos especial atención a la importancia de los elementos extraescolares como los medios de comunicación en la articulación de su discurso narrativo. La importancia de este recurso para la enseñanza de la historia radica en que los sistemas de comunicación han condicionado ciertas formas de transmisión de conocimientos, sobretodo en el estudio de los temas del pasado reciente, los que habitualmente habían estado ausentes de la escuela. Su incorporación busca promover en los jóvenes una mejor comprensión de la sociedad actual y una concepción democrática de “ciudadanía”.

\section{ANÁLISIS Y DISCUSIÓN DE RESULTADOS}

Para cumplir con el objetivo propuesto incluimos en el cuestionario una pregunta donde debían marcar las fuentes de información que les habían servido para obtener sus conocimientos

\footnotetext{
${ }^{1}$ Aprendizajes esperados. Curriculum escolar chileno: $3^{\mathrm{a}}$ Unidad: "El quiebre de la democracia y la dictadura militar" y 4a: "Recuperación de la democracia y transformaciones políticas, sociales y culturales: Chile desde la década de 1990”.

${ }^{2}$ En el tiempo en que se realizó este estudio (2010-2014) el sistema educativo chileno se estructuraba en base a tres tipos de centro escolares según sus fuentes de financiación: colegio particular (CP), de financiación privada; colegio subvencionado (CS) de financiación mixta (concertados) y colegio municipal (CM) de financiación estatal.
} 
sobre el proceso de acceso a la democracia tras el gobierno dictatorial de Pinochet. Debemos especificar que esta pregunta presenta respuestas de carácter acumulativo, debido a que los estudiantes podían escoger más de una opción de respuesta, obteniéndose un total de 1.808 apuntes, de las 598 respuestas válidas, es decir, de los que contestaron la interrogante planteada.

Así, considerando la frecuencia de las respuestas totales de los estudiantes, frente a la pregunta: El tema del paso de una dictadura a una democracia lo conoces a través de... se reconoce que la principal suministradora de conocimientos está entregada por la asignatura de Historia [77,1\%] reconociendo la importancia de la disciplina como fuente para la construcción de conocimiento, situación que se mantiene en el ámbito del aprendizaje formal. Junto a esta alternativa, la segunda opción más votada hace referencia a la familia [75,9\%], lo que determina la influencia de su contexto social más cercano para conocer acerca de los temas históricos recientes. Finalmente, los medios de comunicación presentan un 61,2\% de las opciones válidas, dato no menor si consideramos que estamos insertos en una sociedad de masas y donde los mass media nos bombardean diariamente con informaciones a través de distintos medios (ver Gráfico 1 y Cuadro 1). Esta tendencia se repite cuando se analizan los resultados obtenidos por género, ciudad y tipo de dependencia escolar, como veremos a continuación.

GRÁFICO 1. Fuentes de información sobre el tema del paso de una dictadura a una democracia Recuento de Frecuencia de porcentajes

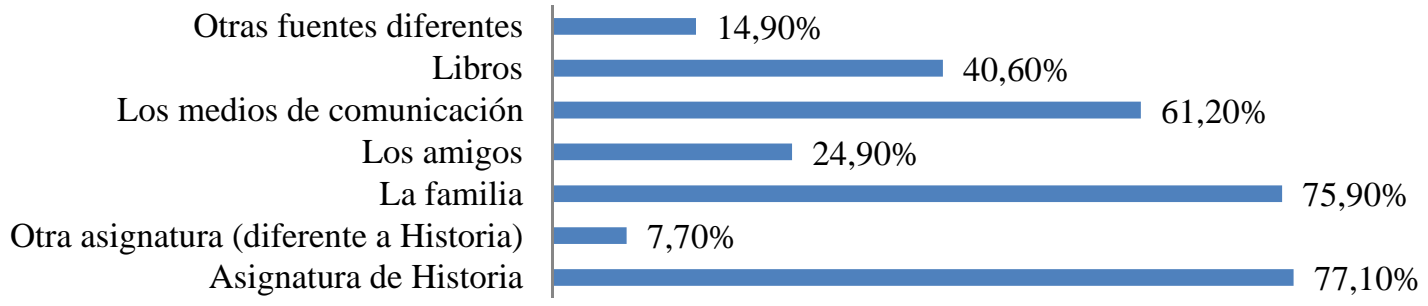

CUADRo I. Fuente de Información sobre el tema del paso de una dictadura a una democracia Recuento de Frecuencia de porcentajes

\begin{tabular}{|c|c|c|c|c|c|c|c|}
\hline & \multicolumn{5}{|c|}{ Casos } & & \\
\hline & \multicolumn{2}{|c|}{ Válidos } & \multicolumn{3}{|c|}{ No responden } & \multicolumn{2}{|l|}{ Total } \\
\hline & $\mathrm{N}^{\mathrm{o}}$ & Porcentaje & $\mathrm{N}^{\mathrm{o}}$ & \multicolumn{2}{|c|}{ Porcentaje } & $\mathrm{N}^{\mathrm{o}}$ & Porcentaje \\
\hline Pregunta & 598 & $97,1 \%$ & 18 & $2,9 \%$ & & 616 & $100,0 \%$ \\
\hline \multicolumn{8}{|c|}{$\begin{array}{l}\text { Frecuencia de respuestas a la pregunta El tema del paso de una dictadura a una democracia lo conoces } \\
\text { a través de.... }\end{array}$} \\
\hline & & & \multicolumn{2}{|c|}{$\mathrm{N}^{\mathrm{o}}$ de respuestas } & \multicolumn{3}{|c|}{$\%$ de alumnos que la marcan } \\
\hline \multicolumn{3}{|c|}{ Asignatura de Historia } & \multicolumn{2}{|r|}{461} & \multicolumn{3}{|r|}{$77,1 \%$} \\
\hline \multicolumn{3}{|c|}{ Otra asignatura (diferente a Historia) } & \multicolumn{2}{|r|}{46} & \multicolumn{3}{|r|}{$7,7 \%$} \\
\hline \multicolumn{3}{|l|}{ La familia } & \multicolumn{2}{|r|}{454} & \multicolumn{3}{|r|}{$75,9 \%$} \\
\hline \multicolumn{3}{|l|}{ Los amigos } & \multicolumn{2}{|r|}{149} & \multicolumn{3}{|r|}{$24,9 \%$} \\
\hline \multicolumn{3}{|c|}{ Los medios de comunicación } & \multicolumn{2}{|r|}{366} & \multicolumn{3}{|r|}{$61,2 \%$} \\
\hline \multicolumn{3}{|l|}{ Libros } & \multicolumn{2}{|r|}{243} & \multicolumn{3}{|r|}{$40,6 \%$} \\
\hline \multicolumn{3}{|c|}{ Otras fuentes diferentes } & \multicolumn{2}{|r|}{89} & \multicolumn{3}{|r|}{$14,9 \%$} \\
\hline \multicolumn{3}{|l|}{ Total } & \multicolumn{2}{|r|}{1808} & & & \\
\hline
\end{tabular}

El análisis por género nos indica respuestas similares entre hombres y mujeres en referencia a la tendencia general antes descrita. Estos datos indican que la asignatura de historia $[\mathrm{M}=79,7 \%$; $\mathrm{H}=74,3 \%]$ es la alternativa con más alta valoración para ambos géneros. A continuación, tanto los 
alumnos como las alumnas señalan la alternativa familia [M=77,4\%; $\mathrm{H}=74,3 \%]$ y, finalmente, tenemos las preferencias correspondiente a los medios de comunicación [M=60,3\%; $\mathrm{H}=62,2 \%$ ], como fuentes informativas para conocer el proceso transitorio, reiterándose la tendencia expuesta en las respuestas totales.

Las respuestas de los estudiantes, analizadas según el tipo de dependencia escolar, nos señalan que las tres opciones mayoritarias se mantienen, pero con algunos aspectos interesantes de destacar con respecto al recuento general. Principalmente, resulta muy llamativo que en el sector particular la familia sea la principal y mayoritaria fuente de información sobre la Transición, [91,5\%] superando con creces a los conocimientos aportados por la disciplina de Historia [69,5\%]. Sin embargo, para los estudiantes de los colegios municipales y subvencionados el ámbito escolar es el principal suministrador de conocimientos sobre esta época reciente. La importante brecha entre los datos entregados por los estudiantes de los centros privados y los suministrados por los centros municipales en relación con el rol desempeñado por la familia [91,5\% frente al 67,4\%], no hace sino poner de manifiesto las diferencias socioculturales de la sociedad chilena, fuertemente segmentada en el ámbito escolar (Belleï, 2004). El resto de las opciones, como por ejemplo los medios de comunicación, se mantiene alrededor de la tendencia general: $[\mathrm{CP}=63,4 \% \mathrm{CS}=61,5 \%$ $\mathrm{CM}=59,6 \%]$.

Por otra parte, las contestaciones de los estudiantes analizadas según las ciudades encuestadas indican, como en el cuadro general, que la asignatura de historia y la familia comparten el protagonismo, siendo ligeramente más frecuente la primera sobre la segunda en los casos de Concepción, Valparaíso y La Serena, y a la inversa en la ciudad de Santiago. Respecto a la familia, destacan los resultados de Concepción [82\%] por sobre sus pares, que también sobresale por los altos valores otorgados a la opción medios de comunicación [CN=74,7\%], frente a los de las otras ciudades que se mantienen bajo la tendencia general [LS=59,6\% VL=55,2\% ST $=53,2 \%]$.

Finalmente, considerando el carácter acumulativo de las respuestas, al mirar el gráfico 2 referente al bloque de respuestas más repetidas, vemos que se frecuentan las opciones mayoritarias antes enunciadas referidas a asignatura de Historia, familia y medios de comunicación, aunque con diferentes formas de relación. Así, si nos centramos en las primeras alternativas en bloque seleccionadas obtenemos: asignatura de historia-familia [9,5\%], asignatura historia- familiamedios de comunicación [9,2\%], asignatura- familia- medios de comunicación- libros [8,5\%] y asignatura de historia [7,4\%].

GRÁFICO 2. Bloques de respuestas más repetidas

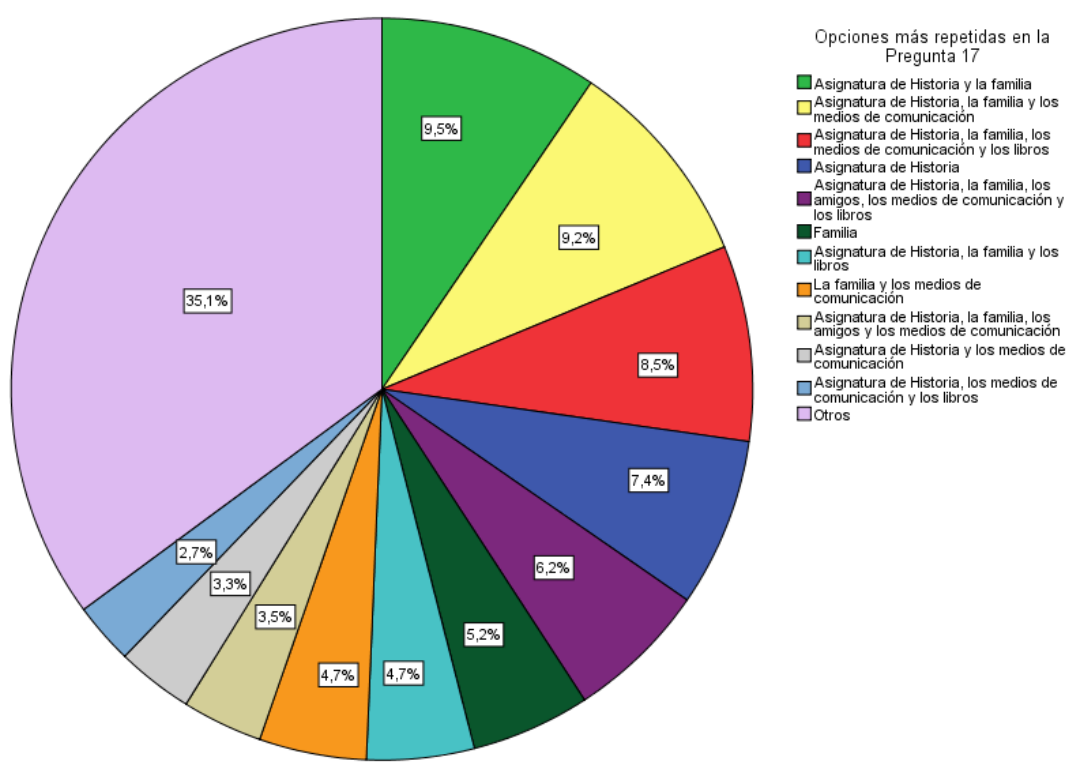




\subsection{Escuela y familia, agentes de conocimientos}

La importancia concedida por los estudiantes a la familia como fuente de conocimiento sobre la historia vivida por las generaciones de más edad pone en evidencia la necesidad de acrecentar la relación familia-escuela para desarrollar el pensamiento histórico en los estudiantes. Es conveniente plantear al alumnado cuestiones que les sean cotidianas, por ejemplo, que escriban su historia de vida, en la que vayan involucrando tanto acontecimientos de su vida personal como de lo que aconteció en su familia o en su ciudad durante el acceso a la democracia; que lleven un objeto familiar que tenga un valor sentimental importante para que narren su historia; que realicen entrevistas para rescatar, así, fuentes que se transmiten de generación en generación y permiten recuperar los testimonios (vivencias y experiencias) de diversos protagonistas. Estos recursos favorecen el conocimiento histórico al proveer información de la memoria colectiva no incluida en los textos impresos, recuperando así elementos de la propia identidad. El uso del testimonio es conveniente para obtener información familiar, local o de diversos temas del siglo XX y la actualidad (Arista Trejo, 2011).

Por ello, no debemos dejarnos abatir frente a las complejas relaciones entre "memoria" e "historia”. Ambas posibilitan un gran desafío al interior del contexto pedagógico y didáctico, referido a procurar instancias de reflexión y de formación democrática de la ciudadanía. Las relaciones presente-pasado facilitan el análisis de la pluralidad y complejidad de los tiempos históricos, aquellos en los que se desarrolla la historia de una sociedad. La incorporación al aula de la contemporaneidad, de la denominada historia del tiempo presente, que no es otra que aquella que viven nuestros alumnos y sus familias, supone la ampliación del concepto de historia, dado que facilita la presencia, cada vez más creciente, de una historia basada en problemas, que facilitaría un acercamiento entre el conocimiento histórico cívico y la realidad socio histórica, fortaleciendo el desarrollo una formación ciudadana crítica y propositiva, permitiéndoles tener un acercamiento a los conocimientos histórico-sociales de manera más constructiva.

En este aspecto, el profesor de Historia cuando enseña un contenido curricular relacionado con la disciplina histórica, debe acentuar la interiorización de valores y actitudes del contexto que forma parte el estudiante, a la vez de considerar la condición de sujetos históricos que poseen sus propios estudiantes, quienes viven en un tiempo y en un espacio determinado que pertenece a una realidad social y cultural, al igual que los sujetos, hechos y procesos y estructuras que estudian (Muñoz, 2016).

Así mismo, "la adhesión de los estudiantes a las narraciones históricas aprendidas fuera de la escuela les conduce a reducir o transformar el contenido del currículum” (Barton, 2010, p. 104) donde el contexto político-social en el que crecieron los jóvenes puede ser determinante en cómo ellos van a concebir la historia. Y, si bien debe existir un respeto hacia esa diversidad, también hay que procurar generar una apertura en la perspectiva de los estudiantes, pues en la escuela no podemos desconocer la diversidad de conocimientos y experiencias que los estudiantes traen consigo desde su entorno más cercano. Esto más que un obstáculo, constituye un enorme desafío a la hora de enseñar la historia del pasado inmediato, pues en el aula se conjugan una diversidad de realidades y experiencias. Al respecto, Eggen y Kauchack (2006) explican que se debe afirmar el compromiso activo del alumno en dar significación a sus experiencias por medio de una enseñanza basada en lo cercano, donde el docente genere este puente cognitivo entre dichas experiencias y la construcción de un aprendizaje significativo en función de los nuevos contenidos que se van integrando. Ello justifica el desafío anteriormente descrito, no obstante no niega la ardua tarea que tiene el docente y con ello el compromiso de la escuela en generar dicho equilibrio. 


\subsection{Los medios de comunicación y la enseñanza de la Transición}

Recordemos que los chicos y chicas encuestados reconocían, casi al mismo nivel que la escuela y la familia [61,2\%], a los medios de comunicación como fuente fundamental de acceso al conocimiento del proceso chileno de transición dictadura-democracia. Este porcentaje de elección es importante si reconocemos que, desde el ámbito pedagógico, la incorporación de los medios de comunicación como recursos didácticos en las aulas todavía es un terreno poco desarrollado. Y nos anima a fortalecer las vinculaciones entre la enseñanza de la Historia y estos recursos de información (televisión, internet, cine de ficción y TICs) como materiales didácticos que sirvan de base para alimentar las estrategias para la enseñanza de la historia, el desarrollo del pensamiento crítico y el fortalecimiento de las habilidades de pensamiento histórico, tales como el análisis de evidencia e interpretación de fuentes, por nombrar algunas.

Estos resultados parecen coincidir, con los obtenidos por el estudio internacional de Educación Cívica y Formación Ciudadana del 2009 (Schulz, et al., 2010), que ha arrojado interesantes datos sobre la influencia de las fuentes extraescolares en la construcción de los discursos de los estudiantes. Por ello, consideramos importante para nuestra investigación analizar cómo influyen estas en las fuentes "oficiales" del discurso escolar establecido dentro del aula de clases y representado por la disciplina de historia. Al respecto, es interesante destacar que los referentes curriculares nacionales han concedido importancia a la libertad de expresión, considerando este valor muy presente en los medios de comunicación, ya que el currículum escolar chileno persigue que los estudiantes valoren los principios de libertad, igualdad, justicia, pluralismo y respeto por los derechos humanos y que reconozcan la legitimidad de diversos puntos de vista para interpretar la realidad (Mineduc, 2013).

La escuela debe sintonizarse con los cambios que se han producido en la sociedad de la información y del conocimiento, para desde ahí hacer frente a las necesidades de una ciudadanía participativa y activa política, social y culturalmente, con el objetivo de poder enfrentar mejor las problemáticas de la historia de su presente. La enseñanza debe tener en cuenta los medios de comunicación que los ciudadanos manejan. Debe conocerlos y usarlos, ya que éstos nos muestran una realidad cambiante, mucho más global e impactante, y nos proporcionan miradas alternativas a las supuestas "verdades objetivas y certeras" que se enseñan en las clases de historia y que predominan en el discurso presente en los libros escolares. Todo esto se plantea con la finalidad de valorar cómo interactúan estos recursos con el aprendizaje para la comprensión de nuestros estudiantes, y así generar experiencias didácticas significativas que desarrollen situaciones de enseñanza-aprendizaje de calidad.

Además, para profundizar más en esta cuestión, en el cuestionario se pidió al estudiantado que especificara el tipo de medios de comunicación a través de los cuales habían obtenido información sobre el proceso de Transición. Al considerar el desglose de las respuestas de opción múltiple vemos que los jóvenes que contestaron destacan como fuentes de información principales para el conocimiento del tema, la televisión [71,5\%], seguida por un porcentaje bastante menor la alternativa internet [25,2\%] (Cuadro 2).

Estos datos, ponen en evidencia que la televisión sigue siendo el medio de comunicación más masivo y que la red de internet cada día va proporcionando mayores alternativas de usos y de significados. Por tanto, los resultados anteriores vendrían a comprobar algunas ideas relacionadas con la percepción de los estudiantes sobre que, fuera de la asignatura de Historia, el ámbito familiar asociado a la televisión constituye el medio fundamental para la adquisición de conocimientos históricos recientes. De esta forma, debemos recalcar que la escuela debe sintonizarse con los cambios que se han producido en la sociedad de la información y del conocimiento, para desde ahí hacer frente a las necesidades de una ciudadanía participativa y activa política, social y culturalmente, y poder así enfrentar mejor las problemáticas de la historia de su presente. 
CuADro 2. Análisis de las opciones de Medios de Comunicación

Recuento y porcentaje de respuestas de alumnos que marcan medios de comunicación

\begin{tabular}{|c|c|c|c|c|}
\hline & & \multicolumn{2}{|l|}{ Frecuencia } & Porcentaje \\
\hline \multirow[t]{3}{*}{ Válidos } & Sí la marcan & \multicolumn{2}{|l|}{366} & 61,2 \\
\hline & No la marcan & \multicolumn{2}{|l|}{232} & 38,8 \\
\hline & Total & \multicolumn{2}{|l|}{$598^{3}$} & 100,0 \\
\hline \multirow{2}{*}{\multicolumn{2}{|c|}{ Medios y su frecuencia en total }} & \multicolumn{2}{|l|}{ Respuestas } & \multirow{2}{*}{$\begin{array}{l}\text { Porcentaje de alumnos en } \\
\text { relación con los } 366 \text { que } \\
\text { escogieron "medios de } \\
\text { comunicación" }\end{array}$} \\
\hline & & $\begin{array}{l}\mathrm{N}^{\mathrm{o}} \text { de } \\
\text { respuestas }\end{array}$ & $\begin{array}{l}\text { Porcentaje en } \\
\text { relación con } \\
\text { el total }\end{array}$ & \\
\hline \multirow[t]{7}{*}{ Medios } & Televisión & 261 & $52,7 \%$ & $71,5 \%$ \\
\hline & Radio & 39 & $7,9 \%$ & $10,7 \%$ \\
\hline & Internet & 92 & $18,6 \%$ & $25,2 \%$ \\
\hline & $\begin{array}{|lll|}\text { Diarios, } & \text { periódicos } & \text { y } \\
\text { revistas } & & \\
\end{array}$ & 27 & $5,5 \%$ & $7,4 \%$ \\
\hline & Películas y documentales & 7 & $1,4 \%$ & $1,9 \%$ \\
\hline & Reportajes y programas & 2 & $0,4 \%$ & $0,5 \%$ \\
\hline & No escriben ningún medio & 67 & $13,5 \%$ & $18,4 \%$ \\
\hline Total & & 495 & $100,0 \%$ & \\
\hline
\end{tabular}

Así mismo, estas respuestas nos recuerdan algunos resultados presentados por una investigación sobre la enseñanza de la sub unidad del curriculum escolar chileno "Régimen militar y Transición a la Democracia” (Magendzo y Toledo, 2009), donde los alumnos encuestados declaran que los recursos para aprender sobre este tema, fuera de la institución escolar, se relacionan con la televisión [69 \%], la conversación con la familia [67\%], los libros [51\%], las películas [48\%] y diarios [30\%], reiterando la importancia de los medios de comunicación como una fuente informativa para aprender sobre lo acontecido en la historia reciente de nuestro país.

Unos y otros resultados ponen en evidencia que la televisión sigue siendo el medio de comunicación más masivo y que la red de internet cada día va proporcionando mayores alternativas de usos y de significados. Esta circunstancia resulta altamente significativa para el ámbito didáctico debido a que, como hemos venido sosteniendo, los medios de comunicación transmiten formas de comportamiento social, haciéndonos ver como normales algunas situaciones presentadas en la pantalla y, a su vez, proporcionando conocimientos e informaciones que permiten la presentación de un sistema cultural determinado y una representación social de la realidad.

En este sentido, recordemos que, para el caso de la Transición chilena, la televisión tuvo un rol fundamental en el convencimiento de los ciudadanos de la necesidad de generar un cambio de gobierno basado en los principios democráticos. Debido a que el bando opositor a Pinochet generó una estrategia publicitaria/televisiva de gran impacto por el mensaje contenida en ella, convirtiéndose en uno de los elementos más importante dentro de la campaña del Plebiscito del 5 de octubre de 1988.

Y por ello, también, esta propaganda política contenida en las franjas televisivas, se ha convertido en una excelente fuente histórica para el estudio del proceso por su cualidad histórica y de material audiovisual (Tironi y Sunkel, 1993) y debiese usarse didácticamente con mayor asiduidad en la enseñanza de la Transición, incorporando su utilización como soporte informativo y como material de estudio desde perspectivas críticas y reflexivas. En relación con ello, debemos recordar el bajo nivel de implementación didáctica de películas y documentales, reconocido por los

\footnotetext{
${ }^{3}$ Las 598 respuestas corresponden a las contestaciones válidas emitidas por los estudiantes. Recordemos que 18 estudiantes no contestaron (ver Cuadro 1).
} 
estudiantes en otra instancia de nuestra investigación, para el aprendizaje de este periodo de la historia reciente (Vásquez, Sánchez-Agustí y Vásquez, 2017).

En síntesis, la enseñanza debe tener presente los medios de comunicación que los ciudadanos tienen, los cuales conforman parte activa de su mundo; debe conocerlos y usarlos, ya que éstos nos muestran una realidad cambiante, mucho más global e impactante, por tanto, nos proporcionan miradas alternativas a las supuestas "verdades objetivas y certeras" que se enseñan en las clases de Historia y que predominan en el discurso presente de muchos de los libros de texto usados en la escuela. Todo esto se plantea con la finalidad de valorar como interactúan estos recursos informativos con el aprendizaje comprensivo de nuestros estudiantes y así generar experiencias didácticas significativas que desarrollen situaciones de enseñanza-aprendizaje basadas en la indagación, contrastación de evidencia y análisis crítico de la información. Además, es importante considerar que el uso de recursos como los medios de comunicación, favorece el aprendizaje a partir del desarrollo de los contenidos procedimentales basados en el método histórico, referidos a la utilización de fuentes e imágenes que se presentan adecuados para su interpretación, generando secuencias basadas en la problematización de los procesos históricos. Esta forma de proceder en el aula potencia la reflexión.

\section{Algunas conclusiones}

Los resultados de nuestra investigación inciden en la vieja idea relacionada con que los aprendizajes de los estudiantes se soportan sobre tres pilares básicos: la escuela, la familia y los medios de comunicación (especialmente la televisión). No obstante, si queremos una educación de calidad resulta particularmente importante encontrar las debidas conexiones pedagógicas y didácticas entre todos ellos, convirtiendo el binomio escuela - entorno en una realidad y no solo en una teoría. Si esto debe aplicarse a toda la enseñanza, resulta imprescindible en el caso de los aprendizajes de los procesos de la Historia Reciente, donde las fuentes familiares constituyen un referente fundamental para su interpretación y donde los medios de comunicación ponen a disposición de los docentes una gran abundancia de fuentes audiovisuales de gran riqueza didáctica ${ }^{4}$.

Por muchas razones la escuela no puede ni debe ocultar dicha influencia ni encerrarse en sí misma para evitarla, siendo conveniente la integración crítica de los medios de comunicación en la educación escolar. Como dos caras de la sociedad democrática, la libertad de comunicación y de expresión tienen que ser equilibradas con una adecuada formación cívica que sepa ponderar y contextualizar sus manifestaciones, ya que la epistemología escolar no puede construirse a contrapelo de la epistemología de la vida cotidiana de los jóvenes, de ahí la necesidad de una educación en los medios (Liceras, 2005). Esta circunstancia resulta altamente significativa para el ámbito didáctico debido a que, como hemos venido sosteniendo, los medios de comunicación educan y transmiten formas de comportamiento y representación social, proporcionándonos conocimientos e informaciones que permiten la presentación de un sistema cultural determinado. Por esta razón, se convierten en una estrategia innovadora de enseñanza de la Historia y de las Ciencias Sociales, por lo cual debiesen usarse didácticamente incorporando su utilización como soporte informativo y como material de estudio, proporcionando actividades que permitan las perspectivas críticas y reflexivas.

Además, es importante considerar que el uso de recursos como los medios de comunicación, favorece el aprendizaje a partir del desarrollo de los contenidos procedimentales, referidos a la utilización de fuentes e imágenes que se presentan adecuados para su análisis e interpretación, permitiendo la generación de secuencias basadas en la problematización de los procesos históricos.

\footnotetext{
${ }^{4}$ A este respecto véase la extensa recopilación de fuentes multimedia sobre los procesos de Transición de Chile y España realizada por el equipo de investigación del proyecto HISREDUC, que se encuentra disponible en http://www5.uva.es/hisreduc/
} 
Por ello, como ya hemos avanzado anteriormente, no debemos dejarnos abatir frente a las complejas relaciones entre memoria e histori”. Ambas posibilitan un gran desafío en la enseñanza de la historia, referido a procurar instancias de debate, argumentación y formación crítica de la ciudadanía, a partir de distintos recursos actuales como son los medios de comunicación, principalmente las fuentes de información que nos ofrece la televisión, internet y los periódicos. Es importante distinguir previamente la pertinencia en el aula de estos elementos, así como definir los criterios que debiesen guiar la selección de los recursos didácticos para tratar estas temáticas. Bajo estas premisas los medios de comunicación se convierten en importantes recursos didácticos a partir de los cuales podemos diseñar estrategias que desarrollen aprendizajes profundos en nuestros estudiantes, pues los medios de comunicación de masas se configuran como una de las influencias más poderosas en la construcción de memorias e identidades (Martínez Rodríguez, 2013).

Desde nuestro punto de vista, se hace necesario un debate de los problemas históricos propios de la Historia Reciente que favorezca el desarrollo de una ciudadanía democrática. Estos contenidos deben estar presentes realmente en el currículo oficial con la finalidad de que sean tratados de una manera relevante dentro de las aulas, y así buscar caminos didácticos para que estas temáticas sean tratadas de una forma significativa en la escuela. Esta integración permitirá superar algunas de las dificultades de una historia aún marcada por un fuerte carácter ideológico, donde coexisten distintas y contrapuestas visiones historiográficas (De Amézola, 2008). Recordemos que es sobre la enseñanza escolar de la Historia donde recae la mayor responsabilidad en la formación cívica de los niños y jóvenes y esta gira particularmente en torno a la comprensión de claves históricas del pasado reciente que permitan explicar el presente y el futuro (Areyuna, 2009). Así la enseñanza de los medios informativos debemos entenderla como una formación en valores y, por consiguiente, tiende a complementar la formación de la ciudadanía y la democracia que se hace en el resto del currículum.

\section{Referencias}

Aceituno, D. (2013). El cine de ficción como lectura crítica de la Historia y su utilización en la Enseñanza: El caso de la Transición a la Democracia en Chile. En J.J. Díaz Matarranz, A. Santisteban y Á. Cascajero (Coords.), Medios de comunicación y Pensamiento Crítico. Nuevas formas de interacción social (pp. 527-538). Guadalajara: Universidad de Alcalá y AUPDCS.

Aparici, R. (2005). Medios de Comunicación y educación. Revista de Educación, 338, 85-99.

Areyuna, B. (2009). Construcción del Pasado, Identidad y Memoria en las narrativas escolares. Una mirada a la Enseñanza de la Historia Reciente en la post Dictadura Chilena. Revista Praxis, 15, 75-82.

Arista Trejo, V. (2011). Cómo se enseña la historia en la educación básica. En. J. Prats, Enseñanza y aprendizaje de la Historia en la Educación Básica. 1a. Ed. México: Editorial Secretaría de Educación Pública.

Arnal, J. (1992). Investigación educativa. Fundamentos y metodologías. Barcelona: Labor.

Aróstegui, J. (2004). La historia vivida. Sobre la historia del presente. Madrid: Alianza.

Barton, K. (2010) Investigación sobre las ideas de los estudiantes acerca de la Historia. Enseñanza de las ciencias sociales, 9, 97-114.

Belleï, C. (2004). Equidad Educativa en Chile: un Debate Abierto. Serie reflexiones: Infancia y adolescencia, 2.

Carretero, M. y Borrelli, M. (2008). Memorias recientes y pasados en conflicto: ¿Cómo enseñar Historia Reciente en la escuela? Cultura y Educación, 20(2), 201-215.

De Amézola, G. (2008). Currículo oficial y memoria. El pasado reciente en la escuela argentina. Enseñanza de las Ciencias Sociales. Revista de investigación, 7, 47-55. 
Eggen, P. y Kauchak, D. (2005). Estrategias docentes. Enseñanza de contenidos curriculares y desarrollo de habilidades de pensamiento. México D.F.: Editorial Fondo de cultura económica.

Figueroa, C. e Iñigo, N. (2010). Reflexiones para una definición de Historia Reciente. En temas y procesos de la historia reciente de América Latina. Santiago: ARCIS CLACSO.

Fontana, J. (2001). La Historia de los hombres. Barcelona: Crítica.

Franco, M. y Levín, F. (2007). La historia reciente en la escuela. Nuevas preguntas y algunas respuestas. Novedades Educativas, 202, 1-5.

Funes, A. (2006). La enseñanza de la historia reciente/presente. Escuela Historia, 5(1), 91-106.

González Gallego, I. (1995). Mensajes, comunicaciones y «mass-media» en los contenidos en Historia. Iber. Didáctica de las Ciencias Sociales, Geografía e Historia, 6, 101-114.

Hernández Cardona, F. X. (2002). Didáctica de las Ciencias Sociales, Geografía e Historia. Barcelona: Graó.

Kriger, M. (2011). La enseñanza de la historia reciente como herramienta clave de la educación política. Narrativas escolares y memorias sociales del pasado dictatorial argentino en las representaciones de jóvenes estudiantes de la Ciudad de Buenos Aires y conurbano (20102011). Revista Persona y Sociedad, XXV(3), 29-52.

Liceras, A. (2005). Medios de comunicación de masas, educación informal y aprendizajes sociales. Iber. Didáctica de las Ciencias Sociales, Geografía e Historia, 46, 109-124.

López Facal, R. y Santidrián V.M. (2011). Los «Conflictos sociales candentes» en el aula. Iber Didáctica de las Ciencias Sociales, Geografía e Historia, 69, 8-20.

Magendzo, A. y Toledo, M. (2009). Educación en Derechos Humanos: currículo historia y ciencias sociales del $2^{\circ}$ año de enseñanza media. Subunidad "Régimen Militar y Transición a la democracia". Estudios Pedagógicos, 35(1), 139-154.

Martínez-Rodríguez, R. (2013). Medios de Comunicación y construcción del conocimiento histórico docente en torno al tema de la Transición Dictadura-Democracia en España. En J.J. Díaz Matarranz, A. Santisteban y Á. Cascajero (Coords.), Medios de comunicación y Pensamiento Crítico. Nuevas formas de interacción social (pp. 175-185). Guadalajara: Universidad de Alcalá y AUPDCS.

Ministerio de Educación de Chile (2005). Informe comisión Formación Ciudadana. Santiago: Editorial MINEDUC.

Ministerio de Educación de Chile (2009). Propuesta Ajuste Curricular Objetivos Fundamentales y Contenidos Mínimos Obligatorios: Historia, Geografía y Ciencias Sociales. Santiago: Editorial MINEDUC.

Ministerio de Educación de Chile (2013). Bases curriculares Historia, Geografía y Ciencias Sociales. $7^{\circ}$ básico a $2^{\circ}$ medio. Santiago: Editorial MINEDUC.

Miralles, P. y Molina, S. (2011). Cómo incorporar el estudio de un acontecimiento, fenómeno o realidad social a la actividad de la clase. En J. Prats (coord.), Geografía e historia. Investigación, innovación y buenas prácticas (pp. 123-138). Barcelona: Graó

Moscovici, S. (1979). El psicoanálisis, su imagen y su público. Buenos Aires, Argentina: Huemul S.A.

Muñoz Labraña, C. (2016). El necesario vínculo entre pasado, presente y futuro. Iber. Didáctica de las Ciencias Sociales, Geografía e Historia, 83, 7-12.

Paz y Miño, J. (2007). Removiendo el presente. Latinoamericanismo e Historia en Ecuador. Quito: Abya Yala.

Rioux, J.P. (1999). La memoria colectiva. En J.P. Rioux y J.F Sirinelli (Dirs.), Para una historia cultural (pp. 341-373). México: Taurus.

Schulz, W., Ainley, J., Friedman, T. y Lietz, P. (2010). Resultados iniciales del Estudio Internacional de Educación Cívica y Ciudadana de la IEA. Amsterdam: Editorial IEA. 
Toledo, I., Magendzo, A., Gutiérrez, V. y Segura, R. (2015). Enseñanza de 'temas controversiales' en la asignatura de historia y ciencias sociales desde la perspectiva de los profesores”. Revista: Estudios Pedagógicos, XLI(1), 275-292.

Vásquez, G., Sánchez-Agustí, M. y Vásquez, N. (2017). Las fuentes de conocimiento histórico sobre la Transición democrática chilena en las aulas de secundaria. CLÍO. History and History teaching, 43, 42-72.

\section{CÓMO CITAR ESTE ARTÍCULO}

Sánchez Agustí, M, Vásquez Leyton, G. y Vásquez Lara, N. (2018). Medios de comunicación e Historia Reciente. Un estudio con alumnado chileno de secundaria. Didáctica de las ciencias experimentales y sociales, 34, 19-34. DOI: 10.7203/DCES.34.10516. 
